\title{
Fast post-Doppler STAP with road map for traffic monitoring: overview and first results
}

\author{
André B. C. da Silva, Stefan V. Baumgartner, and Alberto Moreira \\ Microwaves and Radar Institute, German Aerospace Center (DLR), Oberpfaffenhofen, Germany
}

Correspondence: André B. C. da Silva (andre.silva@dlr.de)

Received: 29 January 2019 - Accepted: 7 July 2019 - Published: 19 September 2019

\begin{abstract}
Synthetic aperture radar (SAR) is an efficient solution for road traffic monitoring due to its high spatial resolution and independence from daylight and weather conditions. In this sense, a number of ground moving target indication (GMTI) algorithms have been developed, whereas their robustness is often achieved with high costs, increased hardware complexity and high computational burden. This paper presents a fast GMTI processor that blends the powerful postDoppler space-time adaptive processing (PD STAP) with an a priori known road map and digital elevation model (DEM). The algorithm presents great potential for real-time processing, decreased hardware complexity and low costs compared to state-of-the-art systems. It is tested using real 4-channel $\mathrm{X}$-band radar data acquired with the DLR's airborne F-SAR.
\end{abstract}

\section{Introduction}

Road traffic monitoring is nowadays a trending topic due to the worldwide increase of road users. Safe and efficient roadway operations require accurate traffic data, whereas detailed traffic information is often available only in the major highways. SAR offers unique image capability for road traffic monitoring by providing high-resolution two-dimensional images (i.e., reflectivity maps) independent from daylight, cloud coverage and weather conditions (Tomiyasu, 1978; Curlander and McDonough, 1991; Moreira et al., 2013). Especially in case of large scale events and catastrophes (i.e., when mobile internet is unavailable and phone communication is impossible), road traffic monitoring with real-time information ensures the safety of the road users and can even save lives.

State-of-the-art GMTI algorithms are available in the literature using a priori knowledge information (Melvin and
Showman, 2006; Bang et al., 2015; Gelli et al., 2017), whereas they often require high hardware complexity and high computational effort. Thus, especially when real-time processing is desired, the use of such methodologies would further increase the complexity of STAP algorithms, which are already very demanding.

This paper presents a fast PD STAP processor that combines a road map with a DEM in order to recognize and to reject false detections, as well as to assign the detected vehicles to their correct position on the roads. Furthermore, accurate velocity and moving direction estimates of the vehicles are obtained. Unlike conventional PD STAP algorithms that process the complete SAR data, the algorithm proposed in this paper uses the road map information for selecting only the relevant data around the roads for processing, thus speeding up the overall processing time significantly.

\section{Signal model and test statistics}

The multi-channel signal model can be expressed by (Ender et al., 2008)

$$
\begin{aligned}
\boldsymbol{s} & =a_{\mathrm{s}} e^{-j \frac{4 \pi}{\lambda} R(t)} D_{\mathrm{t}}(u(t))\left[\begin{array}{c}
D_{\mathrm{r}, 1}(u(t)) e^{j \frac{2 \pi}{\lambda} u(t) x_{1}} \\
D_{\mathrm{r}, 2}(u(t)) e^{j \frac{2 \pi}{\lambda} u(t) x_{2}} \\
\vdots \\
D_{\mathrm{r}, M}(u(t)) e^{j \frac{2 \pi}{\lambda} u(t) x_{M}}
\end{array}\right] \\
& =a_{\mathrm{s}} e^{-j \frac{4 \pi}{\lambda} R(t)} \boldsymbol{d}(u),
\end{aligned}
$$

where $a_{\mathrm{s}}$ is a complex value that accounts for the reflectivity of the scatterer, $\lambda$ is the wavelength, $R(t)$ is the slant range of the target, $D_{\mathrm{t}}(u(t))$ and $D_{\mathrm{r}, m}(u(t))$ are the complex transmitting (TX) and receiving (RX) antenna characteristics of the $m$ th channel, $M$ is the number of RX channels, $x_{m}$ is the 
position of the antenna center in the azimuth direction with respect to the antenna array origin and $\boldsymbol{d}(u)$ is the beamforming or direction-of-arrival (DOA) vector.

The directional cosine $u$ can be expressed by

$u\left(f_{\mathrm{a}}, v_{\mathrm{r}}\right)=\cos \left(\Psi_{\mathrm{DOA}}\right)=\frac{\lambda f_{\mathrm{a}}}{2 v_{\mathrm{p}}}+\frac{v_{\mathrm{r}}}{v_{\mathrm{p}}}$,

where $f_{\mathrm{a}}$ is the Doppler frequency, $v_{\mathrm{r}}$ is the line-of-sight velocity of the target, $v_{\mathrm{p}}$ is the velocity of the platform and $\Psi_{\text {DOA }}$ is the DOA angle of the target measured with respect to the azimuth or flight direction.

The moving target detection is carried out by applying the well-known Adaptive Matched Filter test (Robey et al., 1992)

$\bar{T}\left(r_{k}, f_{\mathrm{a}}\right)=\frac{\left|\boldsymbol{d}^{H}\left(u, f_{\mathrm{a}}\right) \hat{\mathbf{R}}_{W}^{-1}\left(f_{\mathrm{a}}\right) z\left(r_{k}, f_{\mathrm{a}}\right)\right|^{2}}{\boldsymbol{d}^{H}\left(u, f_{\mathrm{a}}\right) \hat{\mathbf{R}}_{W}^{-1}\left(f_{\mathrm{a}}\right) \boldsymbol{d}\left(u, f_{\mathrm{a}}\right)} \lessgtr \eta$,

where $\hat{\mathbf{R}}_{W}$ is the clutter covariance matrix (CCM) estimated empirically from the measured data and $\boldsymbol{z}=\boldsymbol{s}+\boldsymbol{c}+\boldsymbol{n}$ denotes the processed data, where $c$ is the ground clutter or interferences and $\boldsymbol{n}$ is the uncorrelated background white noise. Finally, $\eta$ denotes the desired constant false alarm rate (CFAR) threshold, which is obtained from the heterogeneous ground clutter model introduced in Gierull (2004) and further investigated in Cerutti-Maori et al. (2010) and Gierull et al. (2013).

\section{Proposed processor}

\subsection{Principle}

There are different ways for combining PD STAP with a priori knowledge information (da Silva and Baumgartner, 2016). In particular, this paper presents a PD STAP processor that employs the OpenStreetMap (OSM) (OpenStreetMap, 2018) database for mapping the roads of interest into the multi-channel range compressed (RC) data array, so that only the relevant azimuth samples along the roads are selected for processing. The main objective is to reduce the amount of SAR data to be processed by the computationally expensive PD STAP core, thus decreasing the processing time considerably.

This approach was originally presented for a fast dualchannel processor with real-time traffic monitoring capability (Baumgartner and Krieger, 2012), where the data selected along the roads were processed using only one DOA angle $\Psi_{\text {DOA }}$. The proposed processor is a follow-up version of this fast dual-channel processor, where the data selected along the roads can be optionally processed using multiple DOA angles. The goal of this operation is to increase the number of detections since the radar cross section of the moving target depends strongly on the aspect angle (Bethke et al., 2006).
The principle of the proposed processor is depicted in Fig. 1a, where an airborne SAR illuminates a vehicle moving on a road of interest using multiple DOA angles $\left(\Psi_{\mathrm{DOA}, 1}\right.$, $\left.\ldots, \Psi_{\mathrm{DOA}, n}\right)$ defined within the antenna beamwidth in azimuth (in blue). As the aircraft flies, notice that the moving target presents different DOA angles $\Psi_{\mathrm{DOA}}$ for different instants of time $t$. Figure $1 \mathrm{~b}$ shows the road points mapped into the RC data array for the multiple DOA angles, where the relevant data selected along the road of interest are depicted by the yellow pixels for each DOA angle. In addition, notice that each DOA angle allows obtaining a different portion of the moving target signal (in red) at different instants of time.

\subsection{Structure}

The simplified flowchart of the proposed processor is shown in Fig. 2. The processor works directly on multi-channel RC data (i.e., after pulse compression). First of all, the navigation data of the aircraft are obtained from the inertial measurement unit (e.g., position, velocity, heading and attitude angles: yaw, pitch and roll) as well as the radar system parameters, which are required throughout the complete processing chain.

The Data Selection for Processing block (in green) uses the OSM database for selecting only the relevant SAR data along the roads to be processed by the PD STAP core. Each coherent processing interval (CPI) contains the data selected around one road of interest. Notice that since this operation is carried out at the beginning of the processing chain, the proposed processor can recognize if no roads are contained in the SAR data so that no further data processing needs to be carried out.

The Data Calibration block is essential for detecting the moving targets and for estimating their positions and velocities accurately. The data calibration algorithm corrects not only the offsets due to residual along-track interferometry (ATI) phases and magnitudes of the receive antennas, but also the Doppler centroid over slant range and time by using the attitude angles of the aircraft. Other important parameters (e.g., antenna patterns and along-track baselines) need to be estimated from the measured data only once and stored in the memory in order to speed up the processing time.

The Training Data Selection and CCM Estimation block collects training data free of moving targets and strong discrete signals for the CCM estimation. The quality of the training data impacts directly on the PD STAP performance since the CCM is used for clutter cancellation, so that this operation is of great importance. Some strategies for training data selection and update are presented and discussed in detail in da Silva et al. (2019).

The PD STAP Core is the hearth of the processing chain and has to accomplish three main tasks: (1) the clutter cancellation using the previously estimated CCM $\hat{\mathbf{R}}_{W} ;(2)$ the moving target detection using the previously estimated test statistics $\bar{T}$ and CFAR threshold $\eta$ (cf. Sect. 2); and (3) the 

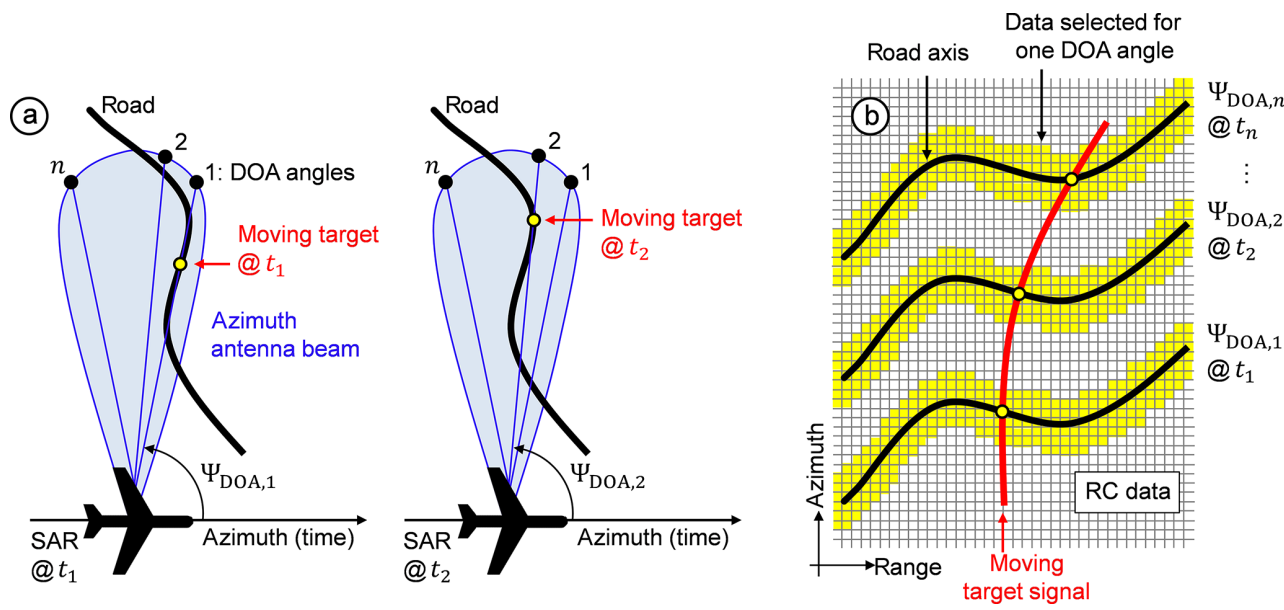

Figure 1. Principle of the proposed processor: (a) acquisition geometry with an airborne SAR illuminating a target moving on the road at different azimuth times $t_{i}$, where each time generally corresponds to a different DOA angle $\Psi_{\mathrm{DOA}, i}$; and (b) the road points mapped into the $\mathrm{RC}$ data array for the multiple DOA angles.

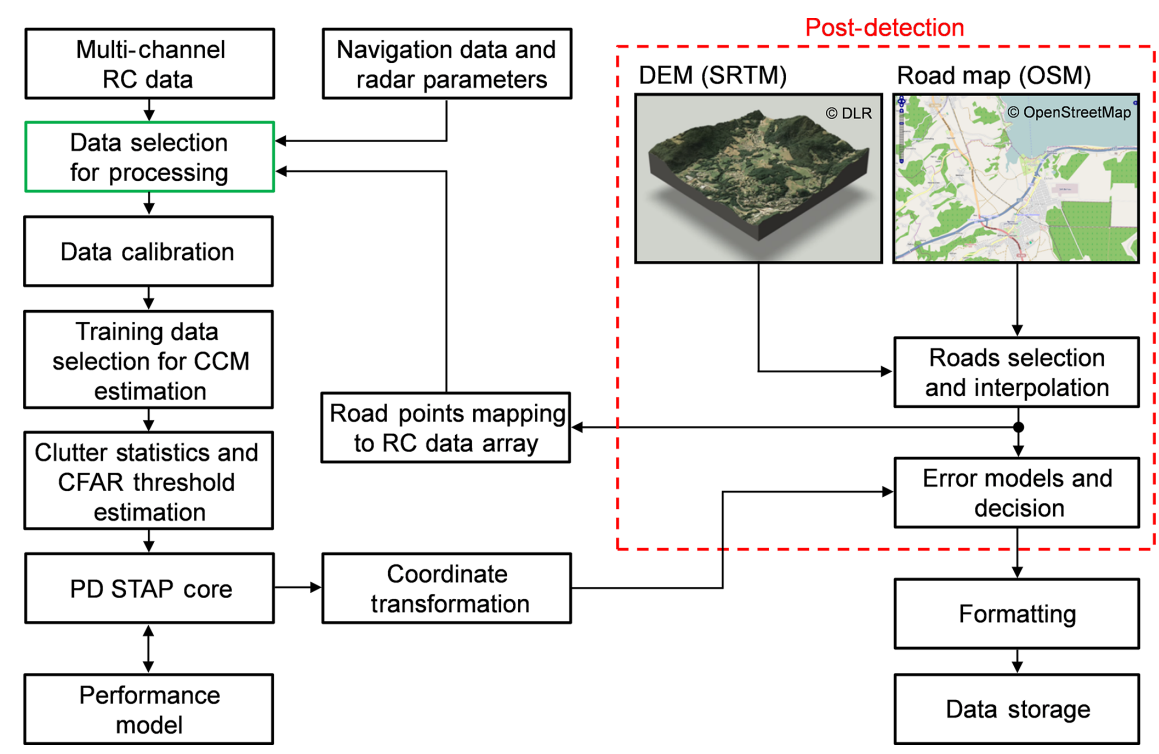

Figure 2. Simplified flowchart of the fast a priori knowledge-based PD STAP processor (top-right image: (C) OpenStreetMap contributors 2019. Distributed under a Creative Commons BY-SA License).

estimation of the position and the slant range velocity of the moving target. The PD STAP is well-known in the literature and the mathematical framework for carrying out these main tasks can be found in (Ender, 1999; Melvin, 2004; Guerci, 2003; Klemm, 2006).

The Performance Model block provides the GMTI performance of the optimum PD STAP processor, including theoretical predictions of the detection performance, blind velocities, minimum detectable velocity (MDV), azimuth positioning error, among others. Some of the performance results were already presented in da Silva and Baumgartner (2017a) based on the framework found in Ender et al. (2008).
In the Coordinate Transformation block, the positions of the moving targets are obtained in both spherical and Universal Transverse Mercator (UTM) Cartesian coordinate systems with respect to the World Geodetic System 1984 (WGS84) reference ellipsoid (i.e., latitude, longitude and elevation).

The Post-Detection module (in red) blends two freely available databases: the road map from the OSM and the DEM from the Shuttle Radar Topography Mission (SRTM) (Rodriguez and et al., 2005; Farr et al., 2007). The DEM is necessary because the OSM does not provide geographical height information. DEMs with better accuracy can also be employed in the future, such as the DEM obtained from the 
German TanDEM-X mission (Krieger et al., 2007; Rizzoli et al., 2017).

The Roads Selection and Interpolation block selects the roads of interest contained in the SAR data and performs an interpolation in order to fill out possible gaps between the OSM road points (Baumgartner and Krieger, 2012). For instance, the proposed processor sets the interpolation distance to the pixel spacing in the slant range direction so that one road point is obtained for each pixel in slant range, as depicted in Fig. 1b.

A decision has to be made for each PD STAP detection in order to verify whether the detection is actually a vehicle moving with high probability on the road or not. This decision is carried out by the Error Models and Decision block, which combines two positioning error models: one for the moving targets (Cerutti-Maori et al., 2008) and one for the OSM road points (da Silva and Baumgartner, 2017b). If the detection is true, then the target is assigned to its closest OSM road point and its absolute velocity on the road and moving direction are estimated (Baumgartner and Krieger, 2012). If the detection lies far from the roads of interest (e.g., a vehicle moving off-road), then it is discarded as a false detection.

In the Formatting block, Keyhole Markup Language (KML) files (Sauerwald, 2013) are generated containing several parameters of the moving targets that can be visualized in Google Earth (2018), such as: spherical and UTM coordinates, velocities, heading directions, Doppler frequencies, among others.

Finally, the traffic data are stored in the mass memory and can be distributed to a traffic management center via data link (Baumgartner et al., 2016).

\subsection{Benefits and limitations}

Some of the main features of the proposed processor are:

1. It is suitable for civilian traffic monitoring, where it is strictly assumed that the vehicles move on known roads;

2. It processes only the relevant azimuth samples selected along the roads instead of the complete SAR data. Thus, the processing time can be considerably decreased, which paves the way for real-time traffic monitoring applications;

3. It is not suitable for areas without road information since the detections obtained from vehicles moving offroad are discarded in the Post-Detection module;

4. It has limited performance for vehicles moving on roads that are parallel or nearly parallel to the flight direction. Indeed, the slant range velocities of such vehicles are generally lower than the MDV and therefore they are suppressed like the stationary ground clutter.

\section{Experimental data}

\subsection{Controlled vehicles}

The proposed processor was firstly tested using real 4channel X-band radar data acquired with the DLR's airborne F-SAR (Horn et al., 2009; Reigber et al., 2013). The flight campaign was conducted over the Allgäu airport in Memmingen, Germany, where five cars with controlled movement and speed were considered. The radar parameters are given in Baumgartner and Krieger (2012). This data set contained $1024 \times 16384$ range-azimuth samples and the probability of false alarm was set to $P_{\mathrm{fa}}=10^{-6}$.

The optical image of the scene is shown in Fig. 3a, where the considered cars (numbered from 1 to 5) and the corner reflector (CR) are indicated. It is pointed out that the cars 1 to 4 moved on the edges of the airport's runway, while car 5 moved off-road in circles. Besides, the runway contained 945 road points from where 128 azimuth samples were selected for each road point. Thus, only a single CPI containing $945 \times$ 128 samples needed to be processed by the PD STAP core (i.e., $0.72 \%$ of the total available data). The selected data for processing are depicted in the yellow box in Fig. 3a.

Figure $3 \mathrm{~b}$ shows the data selected along the runway in Doppler domain before clutter cancellation, where the clutter bandwidth can be seen centered at zero-Doppler. Figure $3 \mathrm{c}$ shows the same data after clutter cancellation, where it can be seen that the region outside of the clutter bandwidth could not be suppressed due to the noise. Figure $3 \mathrm{~d}$ shows the binary map of the detected targets, where the signals from all cars as well as from the CR can be clearly recognized. Although the CR is a stationary target, its signal-to-clutter plus noise ratio (SCNR) exceeds the CFAR threshold for different Doppler frequencies. This response is expected and it visibly differs from the response of a moving target signal.

Figure $3 \mathrm{e}$ shows the final GMTI results, where the false detections were discarded and the true detections from cars 1 to 4 were relocated to the OSM road axis (white line located at the center of the runway). In this figure, the triangles point to the moving directions of the cars and the colors indicate their absolute velocities on the runway. It has to be mentioned that car 5 was discarded as a false detection in the Error Models and Decision block since it moved off-road. The estimated velocities from cars 1 to 4 (car 1 : $10.07 \mathrm{~km} \mathrm{~h}^{-1}$; car 2: $82.52 \mathrm{~km} \mathrm{~h}^{-1}$; car 3: $16.42 \mathrm{~km} \mathrm{~h}^{-1}$; car 4: $44.77 \mathrm{~km} \mathrm{~h}^{-1}$ ) agreed very well with the differential GPS reference data found in Baumgartner and Krieger (2012) with a standard deviation smaller than $5 \mathrm{~km} \mathrm{~h}^{-1}$. Therefore, the proposed processor was able to detect both slow and fast vehicles.

The data selected along the airport's runway (yellow box in Fig. 3a) were processed using only one DOA angle steered at the broadside direction of the antenna array, which was sufficient for detecting all moving targets. Optionally, the selected data could be processed using multiple DOA angles in 

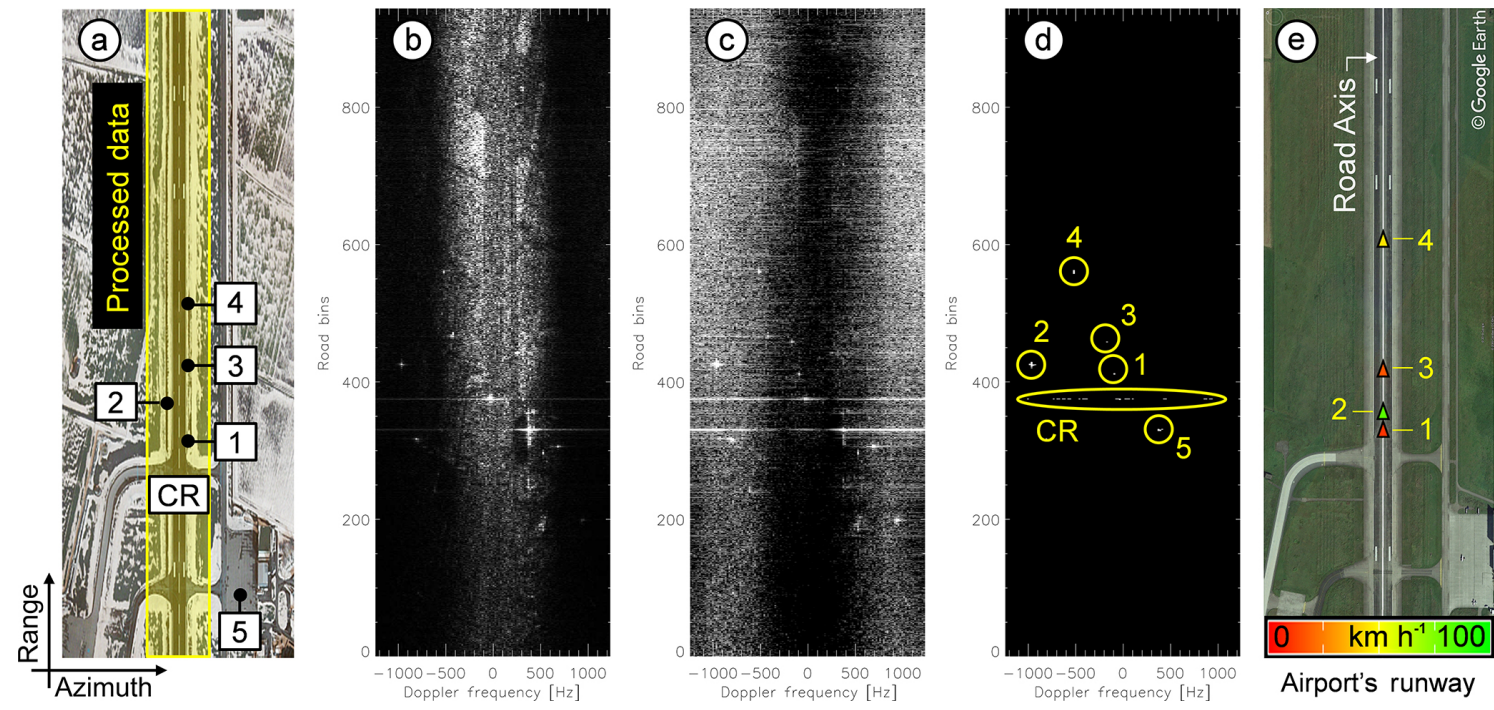

Figure 3. Results from real multi-channel SAR data: (a) optical image of the airport's runway; (b) road-Doppler image before clutter cancellation; (c) road-Doppler image after clutter cancellation; (d) binary detection map (controlled cars numbered from 1 to 5); and (e) final GMTI results.

order to increase the number of moving target detections, as verified in Sect. 4.2.

As a comparison, our former PD STAP processor with a priori knowledge information (Silva and Baumgartner, 2017a) was applied on the same data set, where all the available SAR data were processed. The former processor was also able to detect the cars 1 to 5 , whereas the processing time was nearly 37 times longer than the fast processor proposed in this paper. Indeed, this processing time factor is data dependent and therefore further work is needed considering other data sets with different number of roads and moving vehicles. This evaluation is foreseen for the next experiments with the novel DLR's DBFSAR system (Reigber et al., 2017), which had its maiden flight in November 2016. Anyhow, this result acknowledges the great potential of the proposed processor towards real-time traffic monitoring.

\subsection{Real traffic scenario}

The proposed processor was applied on another 4-channel Xband radar data set acquired with the DLR's airborne F-SAR in the vicinity of Memmingen. This data set contained a section of the highway A7 with several vehicles of opportunity.

Figure 4 shows the final GMTI results obtained by processing the data set using only one DOA angle (steered to the broadside direction of the antenna array axis) as well as using five DOA angles defined within the $3 \mathrm{~dB}$ antenna beamwidth in azimuth, where the probability of false alarm was set to $P_{\mathrm{fa}}=10^{-6}$. The moving vehicles are depicted as colored triangles pointing to their moving directions and the OSM road axes are shown in white. The colors indicate the absolute velocities of the vehicles on the roads and the information box shows examples of parameters that can be displayed for each vehicle.

It is clearly visible that much less detections were obtained by processing the data set using only one DOA angle and some vehicles could not even be detected in this case. Although much more detections can be obtained using multiple DOA angles, this operation can impact on the real-time capability of the system since more computational time is required.

It can also be seen that the proposed processor was able to detect some moving vehicles several times, which reveals the potential of this processor for traffic monitoring applications. It is pointed out that no clustering or tracking algorithms were applied in this experiment for refining the GMTI results.

Unfortunately, no ground truth data were available in this experiment and thus it is not possible to determine the probability of false alarm as well as the errors of the estimated velocities and positions. Even so, the estimated velocities of the vehicles on the highway A7 were reasonable.

To conclude, the GMTI results shown in Fig. 4 demonstrate how an airborne SAR system could be helpful for evaluating the traffic situation in an area of interest (e.g., on the highway A7 towards Ulm and Memmingen). In reality, such traffic data could supplement the information acquired by the road sensors and be used in research for improving traffic models or as information source for road traffic statistics (Leitloff et al., 2010). Furthermore, in some cases (e.g., in rural and primary roads without enough sensor installations or in large scale events and catastrophes) an airborne SAR system could be the only source of traffic data. 


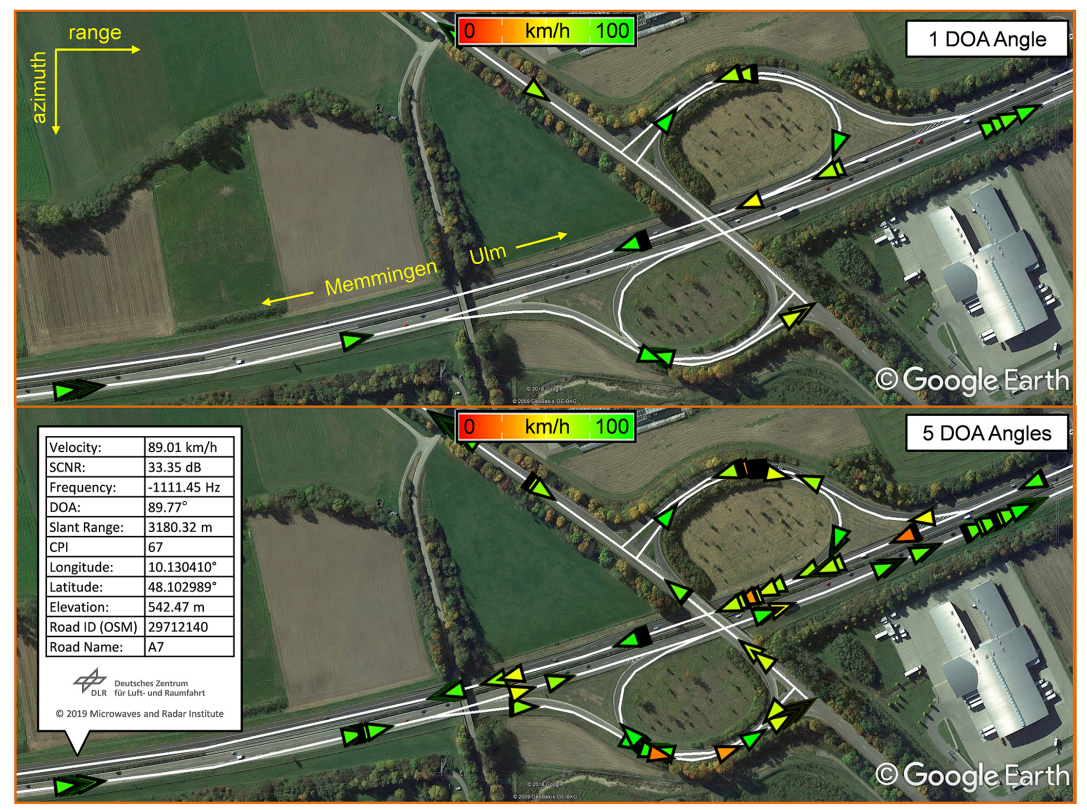

Figure 4. Real traffic on the highway A7 towards Ulm and Memmingen. The vehicles were detected and their parameters were automatically estimated using the proposed fast PD STAP processor considering one and five DOA angles.

\section{Conclusion}

This paper shows the first experimental results obtained with our fast PD STAP processor for real-time traffic monitoring. The proposed processor can detect both slow and fast targets with very good accuracy, and can discard most of the false detections that lie far from the roads of interest. In addition, the processing time is significantly reduced by selecting only the relevant data along the roads to be processed by the PD STAP core. In the controlled experiment with selected vehicles, the proposed processor detected all moving vehicles, being 37 times faster than our former PD STAP processor that processed the complete SAR data. In the experiment with real traffic, the proposed processor detected some moving vehicles several times and much more detections were obtained by processing the selected data using multiple DOA angles. Thus, the potential of the proposed processor for traffic monitoring applications has been verified.

Data availability. The data used within this research project were acquired by DLR's F-SAR airborne radar sensor achieving resolution in the sub-meter range. Therefore, all data products are either restricted to DLR internal evaluation/analyses or subject to export control regulations. In this context, especially the SAR raw data, on which this research is based on, are excluded for public accessibility.

Author contributions. ABCdS contributed on the conceptualization, methodology, software, validation, formal analysis, investigation, writing, visualization, review and editing. SVB contributed on the conceptualization, methodology, validation, formal analysis, visualization, supervision, review and editing. AM contributed on the visualization, supervision, review and editing.

Competing interests. The authors declare that they have no conflict of interest.

Special issue statement. This article is part of the special issue "Kleinheubacher Berichte 2018". It is a result of the Kleinheubacher Tagung 2018, Miltenberg, Germany, 24-26 September 2018.

Acknowledgements. The authors are grateful to the reviewers for their time and effort in revising this paper.

Financial support. This research has been supported by the German Academic Exchange Service (DAAD, grant no. 91557221).

Review statement. This paper was edited by Jens Anders and reviewed by two anonymous referees.

\section{References}

Bang, J., Melvin, W., and Lanterman, A.: Model-based clutter cancellation based on enhanced knowledge-aided parametric covariance estimation, IEEE T. Aero. Elec. Sys., 51, 154-166, 2015. 
Baumgartner, S. V. and Krieger, G.: Fast GMTI algorithm for traffic monitoring based on a priori knowledge, IEEE T. Geosci. Remote Sens., 50, 4626-4641, 2012.

Baumgartner, S. V., Rosigkeit, D., and Nottensteiner, A.: Usability of LTE for Transmitting Radar Data from DLR's Research Aircraft DO 228-212, 36th European Telemetry and Test Conference, Nuremberg, Germany, 181-187, 2016.

Bethke, K. H., Baumgartner, S. V., Gabele, M., Hounam, D., Kemptner, E., and Klement, D.: Air- and spaceborne monitoring of road traffic using SAR moving target indication-Project TRAMRAD, ISPRS J. Photogramm., 61, 243-259, 2006.

Cerutti-Maori, D., Klare, J., Brenner, A. R., and Ender, J. H. G.: Wide-Area Traffic Monitoring With the SAR/GMTI System PAMIR, IEEE T. Geosci. Remote Sens., 46, 3019-3030, 2008.

Cerutti-Maori, D., Gierull, C. H., and Ender, J. H. G.: Experimental verification of SAR-GMTI improvement through antenna switching, IEEE T. Geosci. Remote Sens., 48, 2066-2075, 2010.

Curlander, J. C. and McDonough, R. N.: Synthetic Aperture Radar: Systems and Signal Processing, John Wiley \& Sons, New York, USA, 1991.

da Silva, A. B. C. and Baumgartner, S. V.: A Priori KnowledgeBased STAP for Traffic Monitoring Applications?: First Results, in: European Conference on Synthetic Aperture Radar (EUSAR), 211-215, Hamburg, Germany, 2016.

da Silva, A. B. C. and Baumgartner, S. V.: Novel post-Doppler STAP with a priori knowledge information for traffic monitoring applications: basic idea and first results, Adv. Radio Sci., 15, 77-82, https://doi.org/10.5194/ars-15-77-2017, 2017a.

da Silva, A. B. C. and Baumgartner, S. V.: STAP moving target position estimation accuracy improvement and false detection recognition using a priori road information, International Radar Symposium (IRS), 1-7, Prague, Czech Republic, $2017 \mathrm{~b}$.

da Silva, A. B. C., Baumgartner, S. V., and Krieger, G.: Training Data Selection and Update for Airborne Post-Doppler STAP, IEEE T. Geosci. Remote Sens., 57, 5626-5641, 2019.

Ender, J. H. G.: Space-time processing for multichannel synthetic aperture radar, Electronics \& Communications Engineering Journal, 11, 29-38, 1999.

Ender, J. H. G., Gierull, C. H., and Cerutti-Maori, D.: Improved space-based moving target indication via alternate transmission and receiver switching, IEEE T. Geosci. Remote Sens., 46, 39603974, 2008.

Farr, T., Rosen, P. A., Caro, E., Crippen, R., Duren, R., Hensley, S., Kobrick, M., Paller, M., Rodriguez, E., Roth, L., Seal, D., Shaffer, S., Shimada, J., Umland, J., Werner, M., Oskin, M., Burbank, D., and Alsdorf, D.: The shuttle radar topography mission, Rev. Geophys., 45, 1-33, 2007.

Gelli, S., Bacci, A., Giusti, E.;, Martorella, M., and Berizzi, F.: Effectiveness of Knowledge-Based STAP in Ground Targets Detection with Real Dataset, IET Radar, Belfast, UK, 1-5, 2017.

Gierull, C. H.: Statistical analysis of multilook SAR interferograms for CFAR detection of ground moving targets, IEEE T. Geosci. Remote Sens., 42, 691-701, 2004.

Gierull, C. H., Sikaneta, I., and Cerutti-Maori, D.: Two-step detector for RADARSAT-2's experimental GMTI mode, IEEE T. Geosci. Remote Sens., 51, 436-454, 2013.

Google Earth: available at: https://www.google.com/earth/, last access: 10 November 2018.
Guerci, J. R.: Space-Time Adaptive Processing for Radar, Artech House, Norwood, MA, USA, 2003.

Horn, R., Nottensteiner, A., Reigber, A., Fischer, J., and Scheiber, R.: F-SAR - DLR's new multifrequency polarimetric airborne SAR, International Geoscience and Remote Sensing Symposium (IGARSS), Cape Town, South Africa, 902-905, 2009.

Klemm, R.: Principles of Space-Time Adaptive Processing, The Institution of Engineering and Technology (IET), London, UK, 2006.

Krieger, G., Moreira, A., Fiedler, H., Hajnsek, I., Werner, M., Younis, M., and Zink, M.: TanDEM-X?: A Satellite Formation for High-Resolution SAR Interferometry, IEEE T. Geosci. Remote Sens., 45, 3317-3341, 2007.

Leitloff, J., Hinz, S., and Stilla, U.: Vehicle Detection in Very High Resolution Satellite Images of City Areas, IEEE T. Geosci. Remote Sens., 48, 2795-2806, 2010.

Melvin, W. L.: A STAP overview, IEEE Aero. El. Sys. Mag., 19, 19-35, 2004.

Melvin, W. L. and Showman, G. A.: An approach to knowledgeaided covariance estimation, IEEE T. Aero. Elec. Sys., 42, 10211041, 2006.

Moreira, A., Prats, P.;, Younis, M., Krieger, G., Hajnsek, I., and Papathanassiou, K.: A Tutorial on Synthetic Aperture Radar, IEEE Geoscience and Remote Sensing Magazine, 1, 6-43, 2013.

OpenStreetMap: available at: http://www.openstreetmap.org, last access: 10 November 2018.

Reigber, A., Scheiber, R., Jaeger, M., Prats, P., Hajnsek, I., Jagdhuber, T., Papathanassiou, K., Nannini, M., Aguilera, E., Baumgartner, S., Horn, R., Nottensteiner, A., and Moreira, A.: Veryhigh-resolution airborne synthetic aperture radar imaging: signal processing and applications, P. IEEE, 101, 759-783, 2013.

Reigber, A., Nottensteiner, A., Limbach, M., Jaeger, M., Kosc, A., Scheiber, R., Fischer, J., Di Maria, A., Muller, G., Que, R., Kunemund, M., Trappschuh, K., Pasch, S., Geswein, D., Hoflmayr, D., Gabler, B., Keller, M., Horn, R., and Moreira, A.: DBFSAR: An airborne very high-resolution digital beamforming SAR system, European Radar Conference (EURAD), 175-178, Nuremberg, Germany, 2017.

Rizzoli, P., Martone, M., Gonzalez, C., Wecklich, C., Borla Tridon, D., Bräutigam, B., Bachmann, M., Schulze, D., Fritz, T., Huber, M., Wessel, B., Krieger, G., Zink, M., and Moreira, A.: Generation and performance assessment of the global TanDEM-X digital elevation model, ISPRS J. Photogramm., 132, 119-139, 2017.

Robey, F. C., Fuhrmann, D. R., Kelly, E. J., and Nitzberg, R.: A CFAR Adaptive Matched Filter Detector, IEEE T. Aero. Elec. Sys., 28, 208-216, 1992.

Rodriguez, E., Morris, C. S., Belz, J. E., Chapin, E. C., Martin, J. M., Daffer, W., and Hensley, S.: An assessment of the SRTM topographic products, Jet Propulsion Laboratory (JPL), National Aeronautics and Space Administration (NASA), Pasadena, CA, USA, 2005.

Sauerwald, K.: Einführung in Google Earth und KML, available at: http://landarzar.net/wp-content/uploads/2013/07/KML. pdf (last access: 17 August 2018), 2013.

Tomiyasu, K.: Tutorial Review of Synthetic-Aperture Radar (SAR) with Applications to Imaging of the Ocean Surface, P. IEEE, 66, 563-583, 1978. 